Chirurg 2018 $89: 562$

https://doi.org/10.1007/s00104-018-0641-2

Online publiziert: 4. Mai 2018

(c) Springer Medizin Verlag $\mathrm{GmbH}$, ein Teil von Springer Nature 2018

CrossMark

W. Schröder · C. J. Bruns

Klinik für Allgemein-, Viszeral- und Tumorchirurgie, Universitätsklinik Köln, Köln, Deutschland

\title{
Rekonstruktion der intestinalen Passage durch eine Koloninterposition
}

terposition in $62,3 \%$ bei benignen und in $37,7 \%$ bei malignen Erkrankungen durchgeführt. Das am häufigsten verwendete Kolonsegment war das linke Hemikolon mit 54,2\%, gefolgt vom rechten Hemikolon mit 38,7\% und dem Colon transversum mit 6,9\%. Bei 478 Patienten (25,9\%) erfolgte die Rekonstruktion im hinteren Mediastinum, bei 1156 Patienten $(62,5 \%)$ retrosternal und bei 162 Patienten (8,8\%) subkutan. Die Verwendung des linken im Vergleich zum rechten Hemikolon war mit einer niedrigeren Gesamtmortalität (6,5\% vs. $10,1 \%, p<0,001)$, Morbidität (15,7\% vs. $18,7 \%, p<0,001)$ und Anastomoseninsuffizienzrate $(13,0 \%$ vs. $15,2 \%$, $p<0,001)$ assoziiert. Für die retrosternale Rekonstruktion wurden signifikant niedrigere Komplikations- und Sterblichkeitsraten im Vergleich zur posterioren Hochführung des Kolons berechnet (Morbidität: 9,2\% vs. 18,9\%, $p<0,001$; Mortalität: $4,8 \%$ vs. $7,0 \%, p<0,001)$.

Fazit. Die vorgestellte Metaanalyse ausschließlich retrospektiver Untersuchungen hat trotz aller statistischen Limitationen die gegenwärtig beste Evidenz zu dieser klinisch relevanten Problematik. Die Autoren kommen hier zu dem Schluss, dass für die Rekonstruktion das linke Kolon retrosternal hochgeführt die Therapie der Wahl ist. Die Wahl des Kolonsegmentes ist mit der unterschiedlichen Vaskularisation im rechten und linken Hemikolon gut nachzuvollziehen. Dennoch bleiben auch mit dieser Analyse wichtige Fragen unbeantwortet. Dies betrifft insbesondere die Lokalisation der Anastomose (thorakal vs. zervikal), die wie auch bei der gastralen Rekonstruk- tion Morbidität und Insuffizienzrate erheblich beeinflusst.

\section{Korrespondenzadresse}

Prof. Dr. W. Schröder, FACS, FEBS

Klinik für Allgemein-, Viszeral- und Tumorchirurgie, Universitätsklinik Köln Kerpener Str. 62, 50937 Köln, Deutschland wolfgang.schroeder@uni-koeln.de

Interessenkonflikt. W. Schröder und C. J. Bruns geben an, dass kein Interessenkonflikt besteht. 